\title{
Physical Disabilities in Post-war Jaffna Society - Evidences by Factor and Cluster Analyses
}

\author{
C. Elankumaran
}

Department of Economics, University of Jaffna, Sri Lanka

Copyright $\odot 2019$ by authors, all rights reserved. Authors agree that this article remains permanently open access under the terms of the Creative Commons Attribution License 4.0 International License

\begin{abstract}
This study, which was carried out in 2014, focuses on the nature of physical health conditions in post-war Jaffna society. This is a follow-up study of the original study named "Jaffna Socioeconomic Health Study 1999" (JSEHS-1999). The subjects of the study were husbands or / and wives of both single parent and two parents families. The sample followed in this study is the same as the 1121 families of JSEHS-1999. However, altogether 1036 families from the original study have responded. The original sample drawn in JSEHS was drawn using two-stage stratified sampling technique. An interviewer-administrated questionnaire, similarly prepared with modification to the present situation, was employed to collect follow-up data and information on physical disability measures. In the present study, the general measure of physical fitness or disability employed was "mobility measure" that includes "Walking Conditions" and "Travelling Conditions". The specific measures of physical disabilities were formulated in five dimensions, that are, "Behavior Disability", "Communication Disability", "Personal Care Disability", "Body Disposition Disability" and "Situational Disability". Socio-Economic variables employed to review the background were "Occupational Levels", "Per Capita Income", "Per Capita Expenditure", "Per Capita Energy Consumption" and "Per Capita Protein Consumption". The data collected on these variables were coded or categorized according to the requirement of Statistical Analysis in this study. Exploratory data analysis, Factor analysis, Cluster Analysis, Canonical correlation analysis and Canonical variate analysis were adopted to extract results in this study The walking and travelling conditions of the parents have not shown any adverse effects in this post-war scenario. This study reports, a severe effect in Behavioral Disability which is validated by a major factor "Disability in Self-awareness and knowledge acquisition" of 23\% explanation. Further, this study reports a considerable effect in Communication Disability which is validated by a major factor "Disability in Writing and Symbolic Communication" of $22 \%$ explanation. The effect in
\end{abstract}

Personal Care Disability is minimal, however two major factors "Disability in personal hygiene, clothing and feeding" for husbands and wives respectively shown $20 \%$ and $18 \%$ explanation. It is also reported that moderate effects in Body Disposition and Situational Disabilities are seen validated by two major factors "Disability in Household activity, Dependence \& Environment" for husbands and wives each having $22 \%$ explanations. Valid gender specific disability situations are also reported as found by factor analysis with higher percentage of explanations. Canonical correlation analysis revealed that all the above described disability measures under combined dimensions are highly or moderately correlated which show meaningful disability analysis with validated results. Further, socioeconomic influences are also highlighted in various physical disabilities using Canonical Variate analysis and Cluster analysis to form meaningful clusters of couples or families indicating distinctions by socioeconomic influences.

Keywords Mobility Measure, Behavior Disability, Communication Disability, Personal Care Disability, Body Disposition Disability, Situational Disability, Socioeconomic Impacts, Factor Analysis, Cluster Analysis

\section{Introduction}

In the original study named "Jaffna Socioeconomic Health Study 1999", abbreviated as JSEHS-1999 [1], general health conditions of the people of Jaffna peninsula, had been analyzed and described. Three types of self-assessed health statuses of the parents of the families were the indicators in that assessment. The general self-assessment of their physical, mental, and social health statuses were recorded, analyzed and reported by Elankumaran [2], Elankumaran and Sivagnanasundram [3]. The importance of the original study and a series of this type of follow-up studies is to enable post-war 
development of the region by planners and administrators.

The physical health status expressed by the respondents were later verified and cross-examined by the researcher in detail by 'Physical disability measures'. These disability measures mostly the activities of daily living, were compiled under different dimensions of disabilities and batteries of questions. In the description of the analysis of general physical health status, it was mentioned that detailed studies would be conducted later. Hence the present study deals with the detailed study of 'Physical Health Status'. These are detailed self-assessments and subjected to verifications and cross-examinations and thereby producing more meaningful information from the respondents.

The 'Disability', which includes most of the physical disabilities and some mental disabilities, was first defined and classified by WHO [4]. This is known as ICIDH (International Classification of Impairments, Disabilities, and Handicaps). This includes all types of mental and behavioral disorders. The ICIDH is intended to offer a conceptual framework for information. This is relevant to the long-term consequences of disease, injuries or disorders, and applicable to both personal health care and to the mitigation of environmental and social barriers. The ICIDH basically contains three distinct and independent classifications, each relating to a different plane of experience consequent upon diseases or disorders, which are impairments, disabilities, and handicaps. Figure 1 views the relationships among them. The definitions are also given below.

In the context of health experience, an impairment is any loss or abnormality of psychological, physiological, or anatomical structure or function.

In the context of health experience, a disability is any restriction or lack (resulting from impairment) of ability to perform an activity in the manner or which the range considered normal for a human being.

In the context of health experience, a handicap is a disadvantage for a given individual, resulting from an impairment or a disability, that limits or prevents the fulfillment of a role that is normal (depending on age, sex, etc) for that individual.

Handicap is more problematic. The structure of the handicap classification is radically different from all the disease-related classifications. Circumstances can be expected to place such individuals at a disadvantage in relation to their peers when viewed from the norms of society. Hence, our research concentrates on the intermediate stage 'disability', which is least controversial according to WHO. According to ICIDH of WHO, the disability has been classified and listed under various dimensions. We selected a number of disabilities that are suitable to Jaffna society and compiled them under different headings. The details are given in Table 1.

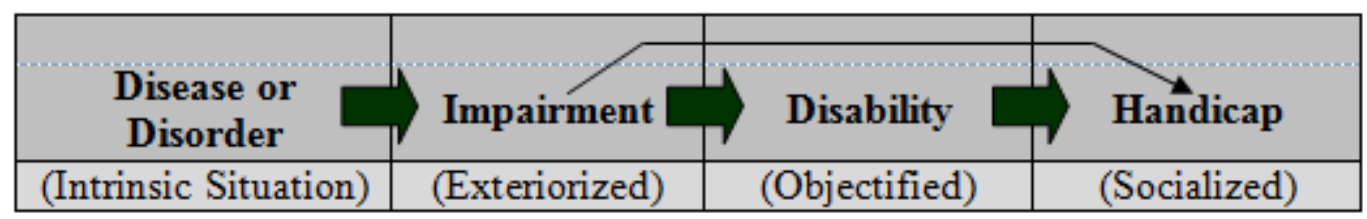

Figure 1. A schematic View of the Classifications of States of 'Disability'.

Table 1. List of Variables Included in the Dimensions of Physical Disabilities of Parents.

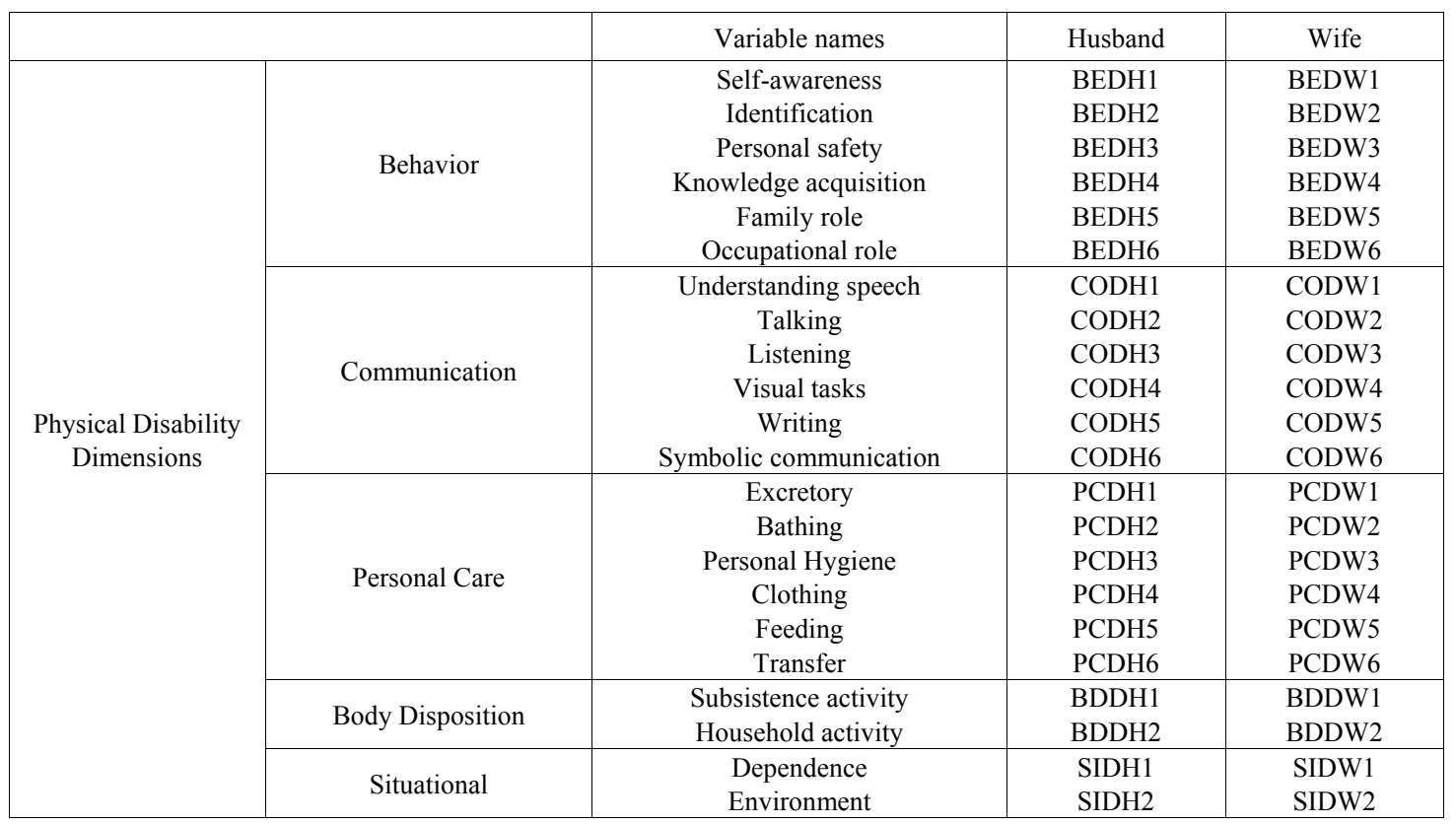




\section{Physical Impacts of War in Jaffna - Objectives and Review}

The overall objective of the original research was focused on the cross-sectional study conducted in the entire population of our study area, which was completely affected by a mass exodus took place in 1995 . The pre exodus situation in the study area was an atmosphere of severe continuous military battles between the security forces and rebels. The post exodus situation was also similar, but a different atmosphere of war existed. Hence the direct impacts of war on the population of the study area is more important for analysis. The direct impacts on the people are of either physical or mental or both.

The study on the effects of war by Sivarajah [5], on the same population which resettled during 1996 and 1997 after the exodus in 1995 gave a clear picture about the physical disabilities of the population and induced to expand the same in this research. This study highlights the physical effects of the population on the basis of 296 patients admitted at the Jaffna general hospital due to war injuries during the one year period from May 1996. About $70 \%$ of the patients were injured due to gun shots, landmines, claymore mines, shell blasts and other related events. It was mentioned in this study that these data did not include the patients treated in the other hospitals, outpatient departments, and private hospitals. Hence the effects would have been more.

The direct war was not existed in the study area at that time. However, the effects prior to and during exodus were more severe and disastrous as the war directly affected the population due to the battles carried out by the security forces on the study area where the study area that was controlled by rebels. There were no official data or studies during the exodus. No other documentary evidence were available to describe the physical impairments due to war in the peninsula.

The focus of this study is deviated from the previous one, as all types of physical impairments and disabilities in terms of activities of daily living including mobility of walking and travelling were taken into consideration in the present study.. This is more meaningful in the sense that, for example, most of the people have been affected on their sight and hearing prior to and during exodus. All activities of daily living as listed in the following section have direct relationships with the atmosphere of war and war related events. These are categorized in general by behavior, communication, personal care, body disposition and situational disabilities.

A small scale study was conducted on a 101 randomly selected civilians by interview regarding war and related events in a small area of the peninsula by Somasundram, et. al. [6]. It was found that $64 \%$ of the persons had developed recognizable psycho-social sequelae. Among them somatization was identified on $42 \%$ of the persons. Similarly PTSD 27\%, Anxiety disorder 6\%, major depression $25 \%$, hostility $19 \%$, relationship problems $13 \%$, alcohol and drug abuse $15 \%$, and functional disability $18 \%$ were reported. All these mental health disorders are the outcome of war and related atmosphere and prove the prevalence of mental disorders in the Jaffna society. This study was conducted during the period between the commencement of large-scale war in 1990 and the exodus took place in 1995. But the severity would have been more after exodus disaster.

Our methodology in the present study is, that we carried out the assessments of physical impairments or disabilities on the parents of the family only. The parents of the nuclear section of the family are younger compared to the parents in the extended section of the family. The physical impairments and disabilities in adults are also naturally age-related problems and hence the old couples of the families were excluded in order to make this research more meaningful. Hence we chose all the 1036 couples available and responded in the sample considered in this follow-up study.

\section{Methodology - Measures, Data \& Statistical Methods}

The present methodology originated from some important disability studies conducted elsewhere. The measures ADL (Activities of daily living) and IADL (Instrumental Activities of Daily Living) are the most common in disability studies by Ebrahim, et. al. [7]; Thorslund, et al., [8]; Norstrom and Thorslund, [9]; Kai, et. al., [10]; and Kempen and Suurmeijer [11]. Our disability measures under different dimensions have been constructed with necessary deletions and modifications of the measures proposed in the above studies. Different dimensions of impairments, disabilities, and handicaps have been proposed to study the severity of physical fitness by McLennan [12] on the basis of WHO [4].

The attitudes and behaviors of humans in epidemiological studies have been analyzed by categorical variables successfully with ordered scores explaining the degrees of severity in the responses related to the attitude or behavior by Janet Reis, [13]. In our study the definitions of the ADL items defined carry ordered categorical scores and hence fit with the international standards. Disability indices are another types of data which are very similar to the ADL items that are introduced in our study due to Jagger, et. al., [14].

The measures of disability, more specifically physical disabilities, have been defined under five different dimensions: Behavior disability, Communication disability, Personal care disability, Body disposition disability, and Situational disability. Each dimension has been further described by a number of disability items, which are measures of Activities of daily living (ADL). In addition, we include an overall dimension called Mobility 
which includes walking conditions and travelling conditions. All the measures of these dimensions are scored by the values from 0 to 5 . The value $\mathbf{0}$ represents 'no disability', 1 represents a 'mild disability', and finally 5 represents a 'severe disability'. The values 2,3 , and 4 represent similar degrees of measures between mild and severe disabilities.

The data related to 'Physical Disability' defined are ordered categorical with values vary from 0 to 5 . With the above description of various data to be used in this paper, the following list of variables were prepared under two sections (Figure 2).

The analysis is begun with Exploratory Data Analysis (EDA) as applied on physical disabilities. Frequency distributions were constructed for all the 22 variables that explain specific physical disabilities for both husbands and wives. The two general physical disability measures of mobility were clearly described by $E D A$.

At the second stage of the analysis, Factor Analysis (FA) was performed individually to all the dimensions. By considering the number of variables included in the dimensions, two dimensions 'body disposition disabilities' and 'situational disabilities' were combined together. In this dimension based analysis the gender was not considered, but gave importance to the disability dimensions only. Further, Factor Analysis was employed on all the dimensions together. Here, the gender was considered separately in order to see any possible gender differences.

In the third stage of the analysis Canonical Correlation
Analysis (CCA) was employed to find out the relationships among the dimensions of physical disabilities. In all the combinations of these analyses, gender was also considered to find out the differences within the dimensions and between genders. In the fourth stage of the analysis the interrelationships of disabilities with socio-economic status was analyzed. Canonical Variate Analysis (CVA) was first employed with the disability variables on the already established [2] socio-economic classes to find out any relationships.

At the final stage of the analysis, Cluster analysis with CVA was employed on the selected socioeconomic variables as listed above on the basis of the clusters formed by disability measures separately. Consequently, nominal logistic regression and ordinal logistic regression were employed on the characterized clusters on the basis of the six selected socioeconomic variables as explanatory variables to explore any relationships.

There are certain limitations in this research. The qualitative responses in a 5-point scale could have been expanded into 7-point or 9-point scale to make the date more sensitive and increase accuracy on the compatibility of high level statistical tools. Sample in the original study was 1121 families drawn using two-stage sampling technique and this sample was followed-up due to the principles of follow-up study. However, due to population increase and restructure over a decade could have slightly changed the increased sample size and responses and thereby little different analytical results.

\section{(a) Physical Disabilities}

\section{(1) Mobility}

(i) Walking condition of Husband

(WACH)

(TRCH)

(ii) Travelling condition of Husband

(WACW)

(iii) Walking condition of Wife

(TRCW)

(2) Behavior Disability

(3) Communication Disability

(4) Personal Care Disability

(5) Body Disposition Disability

(6) Situational Disability

\section{(b) Socio-economic factors}

(1) Occupational level of husband

(2) Occupational Level of Wife

(3) Per Capita Total Expenditure of the family

(4) Per Capita Income/Revenue of family

(5) Per Capita Energy consumption

(6) Per Capita Protein consumption
(OcLeH)

(OcLeW)

(PCExp)

(PCInc)

(PCEnC)

$(\mathrm{PCPrC})$

Figure 2. Dimensions of Disability Study with Explanatory Variables of Socioeconomic Factors 


\section{Results and Discussions on Physical Disabilities}

Altogether 1035 couples were included in the analysis as one of the couples $\left(51^{\text {st }}\right.$ family of $\left.\mathrm{J} 73\right)$ did not respond to this inquiry. As mentioned above the most influential factor 'age' on physical disability has been minimized by selection of the parents of nuclear sections of the families, which are young families compared to the elderly couples in the extended and dependent sections of the families.

\subsection{Mobility}

We first consider the general physical disability which is mobility as explained by walking and travelling conditions of parents. These two characteristics are scored by values from 0 to 5 . Table 2 produces the corresponding frequency distributions. It seems that about $95 \%$ of the husbands and $91 \%$ of the wives are in good walking conditions. About 4\% of the husbands with slight walking disability and $1 \%$ in higher order walking disabilities. Also $9 \%$ of the wives in slight walking disability. Also, there are 2 wives and 2 husbands with severe walking disabilities or unable to walk.

Table 2. The Frequency Distributions of Mobility Items of Husbands and Wives.

\begin{tabular}{|c|c|c|c|c|}
\hline \multirow{2}{*}{ Score } & \multicolumn{2}{|c|}{$\begin{array}{c}\text { Walking (WACH / } \\
\text { WACW) }\end{array}$} & \multicolumn{2}{c|}{$\begin{array}{c}\text { Traveling (TRCH / } \\
\text { TRCW) }\end{array}$} \\
\cline { 2 - 5 } & Husband & Wife & Husband & Wife \\
\hline 0 & $985(95.17)$ & $942(91.01)$ & $977(94.40)$ & $256(24.73)$ \\
1 & $41(03.96)$ & $91(08.79)$ & $38(03.67)$ & $741(71.59)$ \\
2 & $4(00.39)$ & $0(00.00)$ & $6(00.58)$ & $25(02.42)$ \\
3 & $3(00.29)$ & $0(00.00)$ & $8(00.77)$ & $8(00.77)$ \\
4 & $2(00.19)$ & $1(00.10)$ & $6(00.58)$ & $5(00.48)$ \\
5 & $0(00.00)$ & $1(00.10)$ & $0(00.00)$ & $0(00.00)$ \\
\hline
\end{tabular}

Percentages are given within the parenthesis

If we consider the travelling conditions, it seems that about $94 \%$ of the husbands are in good health conditions in terms of cycling. About 4\% of the husbands cannot do cycling and have slight travelling disabilities. Further, the balance $2 \%$ of the husbands has higher order travelling disabilities. If we consider the travelling conditions of wives the interpretation is slightly different as about $25 \%$ of the wives can do cycling and are in higher mobility status. However, about $71 \%$ of the wives, who cannot do cycling, are also in good traveling conditions. Only about 4\% of the wives cannot freely travel and have some difficulties in travelling.

The walking and travelling conditions of the parents have not shown any adverse effects. This may be because these characteristics are usually age related and not much affected in our sample of young couples. Further the war atmosphere would not have given direct effects on these mobility conditions, in fact those effect would have been very low. The two husbands and two wives responded (see the table above) as unable to walk was observed during the survey and this was due to stroke.

\subsection{Individual Disability Dimensions}

The general disability measure 'mobility' described above did not show any effects. However, JSEHS-1999 showed in the same sample that about $61 \%$ of the couples had unsatisfactory physical health status. We shall now concentrate on the specific physical disability measures. We first individually describe the five physical disability dimensions.

\subsubsection{Behavior Disability}

The behavior disability has been described with six characteristics: self-awareness (BED1), identification (BED2), personal safety (BED3), knowledge acquisition (BED4), family role (BED5), and occupation role (BED6). We produced the frequency distributions of these six variables. Tables 3 and 4 give the results for husbands and wives respectively.

Table 3. Frequency Distributions of Behavior Disability Items of Husbands.

\begin{tabular}{|c|c|c|c|c|c|c|}
\hline Score & BEDH1 & BEDH2 & BEDH3 & BEDH4 & BEDH5 \\
\hline 0 & $402(38.84)$ & $757(73.14)$ & $864(83.48)$ & $238(23.00)$ & $870(84.06)$ & $862(83.29)$ \\
1 & $429(41.45)$ & $255(24.64)$ & $148(14.30)$ & $375(36.23)$ & $133(12.85)$ \\
2 & $163(15.75)$ & $21(02.03)$ & $16(01.55)$ & $322(31.11)$ & $24(02.32)$ & $143(13.82)$ \\
3 & $36(03.48)$ & $2(00.19)$ & $5(00.48)$ & $70(06.76)$ & $6(00.58)$ & $16(01.55)$ \\
4 & $5(00.48)$ & $0(00.00)$ & $2(00.19)$ & $27(02.61)$ & $9(00.87)$ \\
5 & $0(00.00)$ & $0(00.00)$ & $0(00.00)$ & $3(00.29)$ & $1(00.10)$ \\
\end{tabular}

Percentages are given within the parenthesis

Table 4. Frequency Distributions of Behavior Disability Items of wives.

\begin{tabular}{|c|c|c|c|c|c|c|}
\hline Score & BEDW1 & BEDW2 & BEDW3 & BEDW4 & BEDW5 \\
\hline 0 & $207(20.00)$ & $683(65.99)$ & $459(44.35)$ & $152(14.69)$ & $702(67.83)$ & $249(24.06)$ \\
1 & $571(55.17)$ & $312(30.14)$ & $548(52.95)$ & $403(38.94)$ & $310(29.95)$ & $724(69.95)$ \\
2 & $211(20.39)$ & $36(03.48)$ & $23(02.22)$ & $337(32.56)$ & $22(02.13)$ & $57(05.51)$ \\
3 & $46(04.44)$ & $4(00.39)$ & $2(00.19)$ & $121(11.69)$ & $0(00.00)$ & $4(00.39)$ \\
4 & $0(00.00)$ & $0(00.00)$ & $2(00.19)$ & $20(01.93)$ & $1(00.10)$ \\
5 & $0(00.00)$ & $0(00.00)$ & $1(00.10)$ & $2(00.19)$ & $0(00.00)$ & $1(00.10)$ \\
\hline
\end{tabular}

Percentages are given within the parenthesis 
The above tables reveal that only about $39 \%$ of the husbands (H) and $20 \%$ of the wives (W) have no disabilities in 'self-awareness'. Further, about $41 \%$ and $55 \%$ of the $\mathrm{H}$ and $\mathrm{W}$ have slight disability and the rest about $20 \%$ and $25 \%$ of $\mathrm{H}$ and $\mathrm{W}$ have severe disability. That is, most of the people, slight and severe, have disturbance of the ability to develop or maintain a mental representation of the identity of the individual's self or body and its continuity over time. Further, disturbance of behavior resulting from interference with conscious-ness or sense of identity and confusion. About $73 \%$ of the husbands and $66 \%$ of the wives have no disabilities in 'identification'. Further, about $25 \%$ and $30 \%$ of the $\mathrm{H}$ and $\mathrm{W}$ have slight disability and the rest about $2 \%$ and $4 \%$ of $\mathrm{H}$ and $\mathrm{W}$ have severe disability. This means that, a considerable number of persons have disturbances of the ability to correctly locate the external objects, understand the events, identify persons, and himself in relation to the dimensions of time and space.

About $83 \%$ of the $\mathrm{H}$ and $44 \%$ of the $\mathrm{W}$ have no disabilities in 'personal safety'. Further about $14 \%$ and $53 \%$ of $\mathrm{H}$ and $\mathrm{W}$ have slight disability and the rest $3 \%$ and $3 \%$ of $\mathrm{H}$ and $\mathrm{W}$ have severe disability. Hence, it is clear that a considerable number of persons, slight and severe, have disturbance of the ability to avoid hazards to the integrity of the individual's body, such as being in hazard from self-injury or from inability to safeguard self from danger. About $23 \%$ of the $\mathrm{H}$ and $15 \%$ of the $\mathrm{W}$ have no disability in 'knowledge acquisition'. But, about 36\%, 39\% slight and $41 \%, 46 \%$ severe disabilities are prevalent. This means, a general disturbance is prevalent on the ability to learn new things, to intellect himself/herself on new skills, and to retain new information.

About $84 \%$ of the $\mathrm{H}$ and $68 \%$ of the $\mathrm{W}$ have no disability in 'family role'. But about $13 \%, 30 \%$ slight and 3\%, $2 \%$ severe disabilities visible. That is, a small group of persons are unable to participate in household activities, to help the spouse on important family matters, to properly behave a parent of the children. About $83 \%$ of the $\mathrm{H}$ and $24 \%$ of the $\mathrm{W}$ have expressed no problems in their 'occupational role'. The percentage for wives seems to be smaller because most of them are housewives and were unable to respond positively. As a result $14 \%, 70 \%$ of $\mathrm{H}$ and $\mathrm{W}$ expressed slight disability and the rest expressed severe disability. Hence we can conclude that, a smaller number of husbands and most of the wives have developed disturbance on the ability to organize and participate in the routine occupational activities. This was suitable for the housewives in the household activities and programs.

We applied factor analysis on all the above twelve variables to explore any structural features among the variables. We extracted four and five factors as suitable. However, the four-factor extraction was more sensible with reality. With the inspection of factor loadings, we were able to interpret the factors as shown in Table 5.

These extracted four factors together explain $70.6 \%$ of the total variation explained by the data. The FA results reveal that about $23 \%$ of the time the behavior disabilities of the persons are prevalent with their self-awareness and knowledge acquisition. The prevalent of behavior disability is about $16 \%$ of the times on their disability in identification. Further the disabilities of personal safety, family role and occupational role of the persons also influence the behavior disability. The prevalent of this disability is about $18 \%$ and $14 \%$ for husbands and wives respectively. Hence we can conclude that the disability in self-awareness and knowledge acquisition of the parents play an important role in the disability of their behavior.

Table 5. The Factors Extracted from the Variables of Behavior Disabilities

\begin{tabular}{|c|l|c|}
\hline Factor & \multicolumn{1}{|c|}{ Name of the Factor } & $\begin{array}{c}\text { Explained } \\
(\%)\end{array}$ \\
\hline 1 & $\begin{array}{l}\text { Disability in Self-awareness and } \\
\text { knowledge acquisition }\end{array}$ & $23.00 \%$ \\
\hline 2 & $\begin{array}{l}\text { Disability in Personal safety \& } \\
\text { family/occupational role of husband }\end{array}$ & $18.30 \%$ \\
\hline 3 & Disability in Identification & $15.50 \%$ \\
\hline 4 & $\begin{array}{l}\text { Disability in Personal safety \& } \\
\text { family/occupational role of wife }\end{array}$ & $13.80 \%$ \\
\hline
\end{tabular}

\subsubsection{Communication Disability}

The communication disability has been described with six characteristics: understanding speech (COD1), talking (COD2), listening (COD3), visual tasks (COD4), writing (COD5), and symbolic communication (COD6). We produced the frequency distributions of these six variables. Tables 6 and 7 present the results for husbands and wives respectively. These tables reveal that, about $66 \%$ of the husbands and $57 \%$ of the wives have no disability in 'understanding speech'. However, about 32\%, 39\% of them with slight and $2 \%, 4 \%$ of them with severe disability prevalent. That is, these persons have loss or restriction of the ability to understand the meaning of verbal messages. About $97 \%$ of the husbands and $96 \%$ of the wives have no disability in 'talking'. This means that only a few people have lost or restricted ability to produce audible verbal messages and to convey meaning through speech.

Table 6. Frequency Distributions of Communication Disability Items of husbands.

\begin{tabular}{|c|c|c|c|c|c|c|}
\hline Score & CODH1 & CODH2 & CODH3 & CODH4 & CODH5 & CODH6 \\
\hline 0 & $681(65.80)$ & $1007(97.29)$ & $930(89.86)$ & $410(39.61)$ & $437(42.22)$ & $287(27.73)$ \\
1 & $327(31.59)$ & $22(02.13)$ & $83(08.02)$ & $250(24.15)$ & $394(38.07)$ & $448(43.29)$ \\
2 & $24(02.32)$ & $4(00.39)$ & $17(01.64)$ & $362(34.98)$ & $134(12.95)$ & $249(24.06)$ \\
3 & $1(00.10)$ & $2(00.19)$ & $4(00.39)$ & $9(00.87)$ & $47(04.54)$ & $35(03.38)$ \\
4 & $0(00.00)$ & $0(00.00)$ & $0(00.00)$ & $4(00.39)$ & $18(01.74)$ & $13(01.26)$ \\
5 & $2(00.19)$ & $0(00.00)$ & $1(00.10)$ & $0(00.00)$ & $5(00.48)$ & $3(00.29)$ \\
\hline
\end{tabular}

Percentages are given within the parenthesis 
Table 7. Frequency Distributions of Communication Disability Items of wives.

\begin{tabular}{|c|c|c|c|c|c|c|}
\hline Score & CODW1 & CODW2 & CODW3 & CODW4 & CODW5 & CODW6 \\
\hline 0 & $593(57.29)$ & $991(95.75)$ & $945(91.30)$ & $487(47.05)$ & $445(43.00)$ & $138(13.33)$ \\
1 & $402(38.84)$ & $41(03.96)$ & $76(07.34)$ & $273(26.38)$ & $375(36.23)$ & $522(50.43)$ \\
2 & $39(03.77)$ & $2(00.19)$ & $10(00.97)$ & $271(26.18)$ & $154(14.88)$ & $319(30.82)$ \\
3 & $0(00.00)$ & $0(00.00)$ & $1(00.10)$ & $3(00.29)$ & $45(04.35)$ & $44(04.25)$ \\
4 & $1(00.10)$ & $0(00.00)$ & $3(00.29)$ & $1(00.10)$ & $11(01.06)$ & $11(01.06)$ \\
5 & $0(00.00)$ & $1(00.10)$ & $0(00.00)$ & $0(00.00)$ & $5(00.48)$ & $1(00.10)$ \\
\hline
\end{tabular}

Percentages are given within the parenthesis

About $90 \%$ of the $\mathrm{H}$ and $91 \%$ of the $\mathrm{W}$ have expressed no disability in 'listening'. But, about $8 \%, 7 \%$ of $\mathrm{H}$ and $\mathrm{W}$ have slight listening problems and about $2 \%, 2 \%$ of $\mathrm{H}$ and $\mathrm{W}$ have severe problems. This means that, this smaller group of people has reduced ability in hearing verbal messages. Some of them expressed that they lost hearing due to bombing and shelling in their neighborhood. About $40 \%$ of the $\mathrm{H}$ and $47 \%$ of the $\mathrm{W}$ have no disability in 'visual tasks'. Further, about $24 \%, 26 \%$ of $\mathrm{H}$ and $\mathrm{W}$ have slight and the rest $36 \%, 27 \%$ of $\mathrm{H}$ and $\mathrm{W}$ have severe vision problems. This reveals that a considerable number of people have lost or reduced ability to execute tasks requiring adequate peripheral vision and activities such as reading, writing, recognition, and visual manipulation. None of the parents are blind, but it was observed that some members of the extended families have blindness or reduced vision due to war related incidents.

About $42 \%$ of the $\mathrm{H}$ and $43 \%$ of the $\mathrm{W}$ have no disability in 'writing'. Further, about 38\%, 36\% have slight and the rest $20 \%, 21 \%$ have severe disability in writing. This severe disability includes completely disabled 5 husbands and 5 wives who cannot even put their signatures in any documents. This completely depended on their literacy and occupation and we did not notice any disability happened due to war effects. About $28 \%$ of the $\mathrm{H}$ and $13 \%$ of the $\mathrm{W}$ have no disability in 'symbolic communication'. Further about $43 \%, 50 \%$ slight and $29 \%, 44 \%$ of $\mathrm{H}$ and $\mathrm{W}$ have severe disability in communicating with symbols. That is, a higher number of persons are not aware of signs and symbols associated with conventional codes and unable to understand and read schematic representation of objects. This may not be a sole reason for literacy, but may be due to the destructed socio-cultural and socio-political environment due to the war.

We now produce the results of factor analysis applied on all the above twelve variables to explore any structural features among the variables. Here also we extracted four and five factors as suitable. However, the five-factor extraction was more sensible with reality. With the inspection of factor loadings, we interpret the factors as shown in Table 8.
Table 8. The Factors Extracted from the Variables of Communication Disabilities

\begin{tabular}{|c|c|c|}
\hline Factor & Name of the Factor & Explained (\%) \\
\hline 1 & $\begin{array}{c}\text { Disability in Writing and symbolic } \\
\text { communication }\end{array}$ & $21.90 \%$ \\
\hline 2 & Disability in Visual tasks. & $12.80 \%$ \\
\hline 3 & $\begin{array}{c}\text { Disability in Talking and listening of } \\
\text { wives }\end{array}$ & $12.60 \%$ \\
\hline 4 & Disability in Understanding speech & $12.10 \%$ \\
\hline 5 & $\begin{array}{c}\text { Disability in Talking and listening of } \\
\text { husbands }\end{array}$ & $11.70 \%$ \\
\hline
\end{tabular}

These extracted five factors together explain $71.1 \%$ of the total variation explained by the data. The FA results reveal that about $22 \%$ of the time the communication disabilities of the persons are prevalent with their disability on writing and symbolic communication. The prevalent of communication disability is about $13 \%$ of the times on their disability in visual tasks and about $12 \%$ of the times with their inability in understanding speech. Further the disabilities of talking and listening of the persons also influence the communication disability. The prevalent of this disability is about $13 \%$ and $12 \%$ for wives and husbands respectively. Hence we can conclude that the disability in writing and symbolic communication of the parents play an important role in the disability of their communication.

\subsubsection{Personal Care Disability}

The personal care disability has been described with six characteristics: excretory (PCD1), bathing (PCD2), personal hygiene (PCD3), clothing (PCD4), feeding (PCD5), and transfer (PCD6). We produced the frequency distributions of these six variables. Tables 9 and 10 give the results for husbands and wives respectively. These tables reveal that, about $79 \%$ of the $\mathrm{H}$ and $83 \%$ of the $\mathrm{W}$ have no difficulties in 'excretory'. But, about 20\%, $16 \%$ slight and $1 \%, 1 \%$ severe excretory difficulties reported. About $88 \%$ of the $\mathrm{H}$ and $92 \%$ of the $\mathrm{W}$ have no disability in 'bathing'. However, about $9 \%, 6 \%$ and $3 \%, 2 \%$ of $\mathrm{H}$ and $\mathrm{W}$ said slight and severe disability in bathing. Hence, a smaller group of persons have bathing problems that is, washing the body and drying self thereafter. 
Table 9. Frequency Distributions of Personal Care Disability Items of husbands.

\begin{tabular}{|c|c|c|c|c|c|c|}
\hline Score & PCDH1 & PCDH2 & PCDH3 & PCDH4 & PCDH5 & PCDH6 \\
\hline 0 & $819(79.13)$ & $917(88.60)$ & $1023(98.84)$ & $1009(97.49)$ & $1027(99.23)$ & $666(64.35)$ \\
1 & $204(19.71)$ & $97(09.37)$ & $9(00.87)$ & $22(02.13)$ & $6(00.58)$ & $326(31.50)$ \\
2 & $5(00.48)$ & $16(01.55)$ & $3(00.29)$ & $2(00.19)$ & $2(00.19)$ & $39(03.77)$ \\
3 & $6(00.58)$ & $5(00.48)$ & $0(00.00)$ & $2(00.19)$ & $0(00.00)$ & $2(00.19)$ \\
4 & $1(00.10)$ & $0(00.00)$ & $0(00.00)$ & $0(00.00)$ & $0(00.00)$ & $2(00.19)$ \\
5 & $0(00.00)$ & $0(00.00)$ & $0(00.00)$ & $0(00.00)$ & $0(00.00)$ & $0(00.00)$ \\
\hline
\end{tabular}

Percentages are given within the parenthesis

Table 10. Frequency Distributions of Personal Care Disability Items of wives.

\begin{tabular}{|c|c|c|c|c|c|c|}
\hline Score & PCDW1 & PCDW2 & PCDW3 & PCDW4 & PCDW5 \\
\hline 0 & $860(83.09)$ & $951(91.88)$ & $1023(98.84)$ & $1027(99.23)$ & $1028(99.32)$ & $772(74.59)$ \\
1 & $164(15.85)$ & $65(06.28)$ & $11(01.06)$ & $5(00.48)$ & $7(00.68)$ \\
2 & $10(00.97)$ & $17(01.64)$ & $1(00.10)$ & $3(00.29)$ & $0(00.00)$ & $240(23.19)$ \\
3 & $0(00.00)$ & $2(00.19)$ & $0(00.00)$ & $0(00.00)$ & $0(00.00)$ & $21(02.03)$ \\
4 & $1(00.10)$ & $0(00.00)$ & $0(00.00)$ & $0(00.00)$ & $0(00.00)$ & $0(00.19)$ \\
5 & $0(00.00)$ & $0(00.00)$ & $0(00.00)$ & $0(00.00)$ & $0(00.00)$ & $0(00.00)$ \\
\hline
\end{tabular}

Percentages are given within the parenthesis

About $99 \%$ of the $\mathrm{H}$ and $99 \%$ of the $\mathrm{W}$ have no disability in performing their 'personal hygiene'. Only $1 \%$ of them has slight disability which includes washing face and hair, care of hands and feet, post-excretion hygiene, dental hygiene and gender specific care. About $97 \%$ of the $\mathrm{H}$ and $99 \%$ of the $\mathrm{W}$ have said they have no disability in 'clothing'. However, the rest $3 \%$ and $1 \%$ have slight disability in clothing themselves. About $99 \%$ of $\mathrm{H}$ and $\mathrm{W}$ have no difficulty in 'feeding'. However, $1 \%$ of them have slight disability in feeding their drinks and foods which includes dispensing beverages, holding drinking and eating utensils, and making food ready etc. Further, about $64 \%$ of the $\mathrm{H}$ and $74 \%$ of the $\mathrm{W}$ have no disability in 'transfer'. Also about $31 \%, 23 \%$ of $\mathrm{H}$ and $\mathrm{W}$ have slight disability and the rest $5 \%, 3 \%$ of $\mathrm{H}$ and $\mathrm{W}$ have severe disability in transfer their body, which includes lying, sitting, standing, and reaching bed, chair etc.

We shall now discuss the results of factor analysis applied on all the above twelve variables to explore any structural features among the variables. In this context too, we extracted four and five factors as suitable. However, the four-factor extraction was more sensible with reality. With the inspection of factor loadings, we were able to interpret the factors as shown in Table 11.

Table 11. The Factors Extracted From the Variables of Personal Care Disabilities

\begin{tabular}{|c|c|c|}
\hline Factor & Name of the Factor & $\begin{array}{c}\text { Explained } \\
(\%)\end{array}$ \\
\hline 1 & $\begin{array}{c}\text { Disability in personal hygiene, clothing and } \\
\text { feeding of husband }\end{array}$ & $20.20 \%$ \\
\hline 2 & $\begin{array}{c}\text { Disability in personal hygiene, clothing and } \\
\text { feeding of wife }\end{array}$ & $18.40 \%$ \\
\hline 3 & $\begin{array}{c}\text { Disability in excretory, bathing and transfer } \\
\text { of husband }\end{array}$ & $17.60 \%$ \\
\hline 4 & $\begin{array}{c}\text { Disability in excretory, bathing and transfer } \\
\text { of wife }\end{array}$ & $17.10 \%$ \\
\hline
\end{tabular}

These extracted four factors together explain $73.3 \%$ of the total variation explained by the data. The FA results reveal that about $38 \%$ of the times the personal care disabilities of the persons are prevalent with their disability on personal hygiene, clothing, and feeding. Further, about $35 \%$ of the times the personal care disabilities of the persons are prevalent with their disability on excretory, bathing, and transfer.

\subsubsection{Body Disposition and Situational Disabilities}

The body disposition disability has been described with two characteristics: subsistence activity (BDD1) and household activity (BDD2). We produced the frequency distributions of these two variables. Table 12 gives the results for husbands and wives. This table reveals that about $80 \%$ of the $\mathrm{H}$ and $82 \%$ of the $\mathrm{W}$ have no disability in 'subsistence activity'. Further about 18\%, 17\% slight and the rest $2 \%, 1 \%$ severe disability in subsistence activities reported which includes shopping in the immediate neighborhood, preparing and serving food, cleaning the utensils, etc. About $34 \%$ of the $\mathrm{H}$ and $59 \%$ of the $\mathrm{W}$ have no disability in 'household activity'. Also about 59\%, 38\% of $\mathrm{H}$ and $\mathrm{W}$ and the rest $7 \%, 3 \%$ of $\mathrm{H}$ and $\mathrm{W}$ have slight and severe disability in household activities which includes washing cloths, helping children, cleaning the house and compound etc.

The situational disability has been described with two characteristics: dependence (SID1) and environment (SID2). We produced the frequency distributions of these two variables. Table 13 gives the results for husbands and wives. This table reveals that about $51 \%$ of the $\mathrm{H}$ and $61 \%$ of the W have no disability in 'dependence'. About $18 \%$, $17 \%$ of $\mathrm{H}$ and $\mathrm{W}$ and the rest $31 \%, 22 \%$ of $\mathrm{H}$ and $\mathrm{W}$ have slight and severe disability in dependence which includes circumstantial dependence such as existence and activity upon life-sustaining equipment and medicine, special diet, special care, etc. About $63 \%$ of the $\mathrm{H}$ and $64 \%$ of the W have no disability in 'environment'. Further, about 31\%, $29 \%$ of $\mathrm{H}$ and $\mathrm{W}$ have slight disability and the rest $6 \%, 7 \%$ of $\mathrm{H}$ and $\mathrm{W}$ have severe environmental disabilities. This includes disabilities relating to climatic conditions, noise, tolerance of illumination, tolerance of work stresses, and other environmental factors. 
Table 12. Frequency Distributions of Body Disposition Disability Items of Husbands and Wives

\begin{tabular}{|c|c|c|c|c|}
\hline \multirow{2}{*}{ Score } & \multicolumn{2}{|c|}{ Husband } & \multicolumn{2}{|c|}{ Wife } \\
\cline { 2 - 5 } & BDDH1 & BDDH2 & BDDW1 & BDDW2 \\
\hline 0 & $830(80.19)$ & $349(33.72)$ & $849(82.03)$ & $175(16.91)$ \\
1 & $184(17.78)$ & $616(59.52)$ & $10(00.97)$ & $394(38.07)$ \\
2 & $16(01.55)$ & $61(05.89)$ & $0(00.00)$ & $31(03.00)$ \\
3 & $2(00.19)$ & $5(00.48)$ & $0(00.00)$ & $0(00.00)$ \\
4 & $3(00.29)$ & $4(00.39)$ & $1(00.10)$ & $1(00.10)$ \\
5 & $0(00.00)$ & $0(00.00)$ & \\
\hline
\end{tabular}

Percentages are given within the parenthesis

Table 13. Frequency Distributions of Situational Disability Items o Husbands and Wives

\begin{tabular}{|c|c|c|c|c|}
\hline \multirow{2}{*}{ Score } & \multicolumn{2}{|c|}{ Husband } & \multicolumn{2}{|c|}{ Wife } \\
\cline { 2 - 5 } & SIDH1 & SIDH2 & SIDW1 & SIDW2 \\
\hline 0 & $532(51.40)$ & $652(63.00)$ & $628(60.68)$ & $295(64.06)$ \\
1 & $230(22.22)$ & $317(30.63)$ & $136(13.14)$ & $69(06.67)$ \\
2 & $238(23.00)$ & $56(05.41)$ & $13(01.26)$ & $6(00.58)$ \\
3 & $30(02.90)$ & $9(00.87)$ & $0(00.00)$ & $2(00.19)$ \\
4 & $5(00.48)$ & $1(00.10)$ & $0(00.00)$ & $0(00.00)$ \\
5 & $0(00.00)$ & $0(00.00)$ & & \\
\hline
\end{tabular}

Percentages are given within the parenthesis

Table 14. The Factors Extracted from the Variables of Body Disposition and Situational Disabilities

\begin{tabular}{|c|c|c|}
\hline Factor & Name of the Factor & Explained (\%) \\
\hline 1 & Disability in household activity, dependence, \& environment of wife & $22.4 \%$ \\
2 & Disability in household activity, dependence, \& environment of husband & $22.3 \%$ \\
3 & Disability in Subsistence activity & $18.8 \%$ \\
\hline
\end{tabular}

Finally we discuss the results of the factor analysis applied on the combined dimensions of body disposition and situational with all the eight variables. Here we extracted three factors. With the inspection of factor loadings, we were able to interpret the factors as shown in Table 14.

These extracted three factors together explain $63.4 \%$ of the total variation explained by the data. The FA results reveal that about $45 \%$ of the times the disabilities of the persons are prevalent with their disability on household activity, dependence and environment. Further, about 19\% of the times the disabilities of the persons are prevalent with their disability on subsistence activity.

\subsection{Overall Physical Disabilities}

In the above section we discussed about the physical disabilities by dimensions, where we did not consider the gender effects within the dimensions. However, we found that some of the extracted factors are gender specific. We shall now consider the entire gender-specific-physical disability space and try to find out the common causes associated with gender. Thus, all the 22 variables for husbands were considered separately and similarly for wife.
Again factor analysis was suitable. We first employed FA on the 22 physical disability variables of husbands and then for wives. The extracted factors and their factor loadings for husbands and wives are not produced to minimize the length of this paper.

The inspection of factor loadings, enable us to extract eight factors, which are given in Table 15 and 16 . We examined 7, 8, 9, and 10 factors, but we found that 8-factor formation was sensible for both husbands and wives. All the eight factors for husbands together explain $72.5 \%$ of the total variation and similarly all the eight factors for wives explain $68.6 \%$ of the variation. These two tables reveal that the extracted eight disability factors have been formed by a more meaningful manner and it is also slightly different from the factor formations within the dimensions considered with their spouses. The gender difference in physical disabilities could be compared if we consider the formations of factors for husbands and wives. If we examine the factors as shown by both of these tables, it is clear that almost all the factors are identical except some minor differences regarding disabilities in understanding speech and subsistence activity. Further, the percentages of explanations of the factors are also slightly different. 
Table 15. The Factors Extracted from the Physical Disability Variables for Husbands.

\begin{tabular}{|c|c|c|}
\hline Factor & Name of the Factor & Explained (\%) \\
\hline 1 & Disability in self-awareness, knowledge acquisition, understanding & $14.4 \%$ \\
& speech, writing, and symbolic communication & $12.2 \%$ \\
3 & Disability in excretory, bathing, transfer, and household activity & $11.6 \%$ \\
4 & Disability in personal hygiene, clothing and feeding & $10.4 \%$ \\
5 & Disability in identification, personal safety, family role & $06.7 \%$ \\
6 & and occupational role & $06.6 \%$ \\
7 & Situational Disability & $05.8 \%$ \\
8 & Disability in talking and listening & $04.9 \%$ \\
\hline
\end{tabular}

Table 16. The Factors Extracted from the Physical Disability Variables for Wives.

\begin{tabular}{|c|c|c|}
\hline Factor & Name of the Factor & Explained (\%) \\
\hline 1 & Disability in self-awareness, knowledge acquisition, & $13.0 \%$ \\
& writing, and symbolic communication & $11.8 \%$ \\
3 & Disability in excretory, bathing, transfer, and household activity & $10.3 \%$ \\
4 & Disability in personal hygiene, clothing and feeding & $07.8 \%$ \\
& Disability in identification, understanding speech, & $07.2 \%$ \\
6 & and subsistence activity & $06.8 \%$ \\
7 & Disability in personal safety, family role, and occupational role & $06.7 \%$ \\
8 & Situational Disability & $05.1 \%$ \\
\hline
\end{tabular}

\section{Relationships among the Dimensions of Disabilities}

The relationships between all the physical disability dimensions included in the list of variables in Section 3 are described in this section. Canonical correlation analysis (CCA) was employed for this purpose.

We shall first consider the relationships among the four physical disability dimensions: 'Behavior Disability' (BED), 'Communication Disability' (COD), 'Personal Care Disability' (PCD), and 'Body Disposition and Situational Disabilities' (BSD). As in the previous section here also we combined the last two dimensions. We consider all combinations of the relationships between husbands and wives on all the dimensions. Table 17 describes the first canonical correlations of all the possible combinations.

The following table reveals that the behavior disability of husband is highly and reasonably correlated with his all other physical disabilities and reasonably associated with his wife's behavior and communication disabilities. Similarly, wife's behavior disability is highly and reasonably correlated with her communication disability and her husband's communication, body disposition, and situational disabilities. In addition, the husbands and wives seem to have reasonable common communication, body disposition, and situational disabilities. Further, it is also clear that individually their personal care and body disposition and situational disabilities are reasonably correlated.

We investigated further the above highlighted relationships with the help of standardized canonical coefficients. It is clear that the associations of physical disabilities between husbands and wives are mainly due to their common disabilities in identification, knowledge acquisition, symbolic communication, and subsistence activities. If we consider the associations of different disabilities of husbands, it is clear that the disability in knowledge acquisition further enables the disability in symbolic communication. The husbands' disabilities in personal safety and personal hygiene are interrelated. His family role is related to his subsistence activities (minor household activities). Further, his body transfer and household activities are also interrelated. Regarding the associations of wife's disabilities, we may conclude that knowledge acquisition disability causes the disabilities in symbolic communication. Transfer and household activities are also interrelated 
Table 17. First Canonical Correlations among Physical Disability Dimensions between Husbands and Wives.

\begin{tabular}{|c|c|c|c|c|c|c|c|c|c|}
\hline \multirow{2}{*}{\multicolumn{2}{|c|}{ First CC }} & \multicolumn{2}{|c|}{ BED } & \multicolumn{2}{|c|}{ COD } & \multicolumn{2}{|c|}{ PCD } & \multicolumn{2}{|c|}{ BSD } \\
\hline & & \multirow{2}{*}{$\begin{array}{c}\text { Husb } \\
--- \\
\end{array}$} & \multirow[t]{2}{*}{ Wife } & \multirow[t]{2}{*}{ Husb } & \multirow[t]{2}{*}{ Wife } & \multirow[t]{2}{*}{ Husb } & \multirow[t]{2}{*}{ Wife } & \multirow[t]{2}{*}{ Husb } & \multirow[t]{2}{*}{ Wife } \\
\hline \multirow[b]{2}{*}{ BED } & Husb & & & & & & & & \\
\hline & Wife & 0.75 & --- & & & & & & \\
\hline \multirow[b]{2}{*}{ COD } & Husb & 0.80 & 0.52 & --- & & & & & \\
\hline & Wife & 0.58 & 0.73 & 0.67 & --- & & & & \\
\hline \multirow[b]{2}{*}{ PCD } & Husb & 0.57 & 0.45 & 0.44 & 0.36 & --- & & & \\
\hline & Wife & 0.24 & 0.43 & 0.26 & 0.36 & 0.41 & --- & & \\
\hline \multirow{2}{*}{ BSD } & Husb & 0.53 & 0.52 & 0.41 & 0.32 & 0.61 & 0.29 & --- & \\
\hline & Wife & 0.35 & 0.41 & 0.30 & 0.32 & 0.31 & 0.60 & 0.57 & --- \\
\hline
\end{tabular}

\section{Socio-Economic Influences on Physical Disability}

In JSEHS-1999, we exhibited the prevalence of physical, mental, and social health statuses and their possible relationships with socio-economic status. We found the existence of four groups of couples with different self-assessed health conditions. In the preceding sections of this paper we analyzed in details about the physical disabilities and established some relationships between various dimensions and variables. Now we shall concentrate on the possible socio-economic influence on the physical health conditions.

With the outcome of our analyses of socio-economic status in JSEHS-1999 [2], we selected some key socio-economic variables for this purpose. The key socio-economic variables are; 'occupation of parents', 'per capita income', 'per capita expenditure', 'per capita energy consumption', and 'per capita protein consumption'. Altogether 1034 couples (cases) were included in the analysis. Working with higher number of variables become statistically cumbersome. Hence, in order to reduce the dimensionality of our problem, we applied CDA on all the variables by dimensions and gender separately and then by the whole lot of variables. But, it was not possible to find out the variables, which are least important and less influential in discrimination. Hence, we concluded that all disability variables are equally important.

The purpose of this investigation was to find out the influence of any of our disability measures on these four groups. We applied CDA on all the variables by dimensions and gender separately and then by the whole lot of variables with the four socio-economic groups. We found that the present combinations of physical disability or mental disorder measures do not have any direct influence on the socio-economic groups. Hence we wanted to approach the problem in a different way. Therefore, we wanted to find out distinct disability groups of couples on the basis of the disability measures in combinations and then interrelate with some selected socio-economic variables.

As mentioned above, we wanted to know the clusters of couples, which have distinct physical disabilities. By considering the number of variables and similarities of dimensions, we combined behavior and communication disabilities together and personal care, body disposition, and situational disabilities together. We included the variables of husbands and wives together as our focus is on families. We applied our usual hierarchical cluster analysis with Ward's minimum variance method to find out the different clusters of couples, which possess distinct features of physical disabilities. We then applied CDA to characterize the features of the clusters of couples.

\subsection{Influence on Behavior and Communication Disabilities}

We first consider the case of behavior and communication disabilities which are closely interrelated. Figure 3, describes the dendrogram of the couples for the combined disabilities. This figure clearly reveals that there are two distinct groups of couples who possess different behavior and communication disabilities. To identify the features of the above two clusters, we employed CDA. In fact, the second variate is redundant as the first variate explains $100 \%$ of the variation. With the help of linear discriminant analysis, we confirmed that $89 \%$ of the observations were correctly classified. 


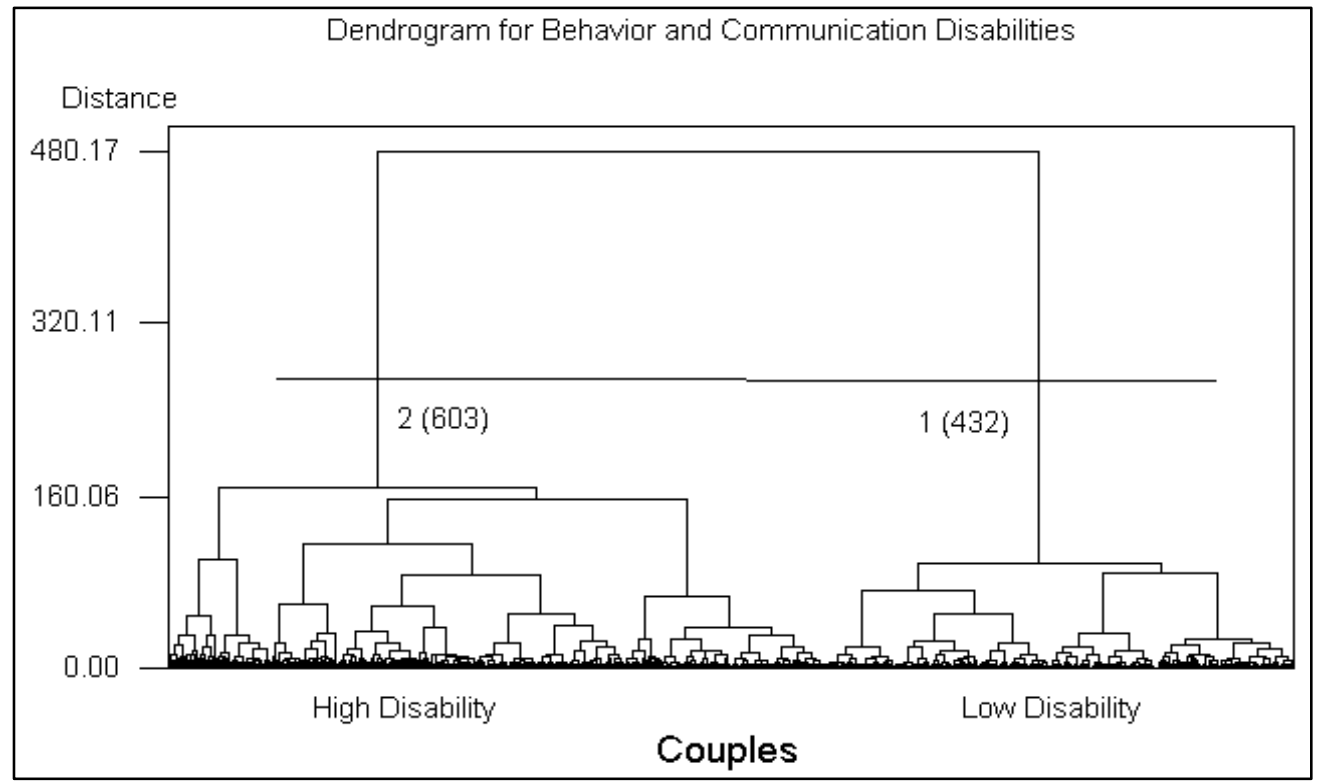

Figure 3. Dendrogram Showing the Clusters of Couples for Behavior and Communication Disabilities.

We inspected the pooled within class standardized canonical coefficients of the CDA to identify the features of these clusters. We found that the cluster 2 of $58 \%$ of the couples is influenced by the higher degree of disabilities in self-awareness, knowledge acquisition, understanding speech, and symbolic communication of husbands and in identification, understanding speech, and listening of wives. The incidences of these characteristics are low in the cluster 1 of about $42 \%$ of the couples. Other disabilities do not seem to dominate in the discrimination of these two clusters. Hence we could name the clusters 1 and 2 as 'Couples of low disability in behavior and communication' and 'Couples of high disability in Behavior and Communication' respectively.

We employed CDA on the two clusters with the six socio-economic variables mentioned above as predictors. We found that the selected variables clearly discriminating the two groups. As discussed above, the second canonical variate is redundant. To explore the influences of the six socio-economic variables on these clusters we inspected the standardized canonical coefficients. We found that higher occupational level of husbands is strongly associated with the cluster of the couples of low disabilities in behavior and communication. Higher occupational level of wives seems to have reasonable association with this cluster of couples. Further, higher per capita income and expenditure also seem to have average association with this cluster. Food consumption seems to have no effect on the disability in behavior and communication. Hence we can conclude that higher behavior and communication disabilities are prevalent in the people of low occupation and low monetary status. This is true in about $58 \%$ of the couples.

\subsection{Influence on Personal Care, Body Disposition and Situational Disabilities}

We now consider the case of personal care, body disposition, and situation disabilities, which all are interrelated. Figure 4, describes the dendrogram of the couples for the combined disabilities.

This figure reveals that the number of clusters must be between two and five. However, we fixed the number of clusters be three with the aid of linear discriminant analysis, which provided the maximum correct classification $95 \%$. We applied CDA on these three clusters with the corresponding disability measures and confirmed about the existence of three clusters. The first two canonical variates together explain $100 \%$ of the variation.

We also inspected the pooled within class standardized canonical coefficients to identify the distinct features of these clusters. The coefficients show that the cluster 2 has no influence from any of the variables. Further, the cluster 3 has been discriminated by higher values of CV2 and low values of CV1 and hence majority of the variables influences the cluster. We also used cluster wise descriptive statistics to identify the features of the clusters as shown in Table 18 . This table reveals that about $20 \%$ of the people have higher and about $17 \%$ of the people have average personal care and related disabilities. The rest $63 \%$ of the people have mild or no personal care and related disabilities. 


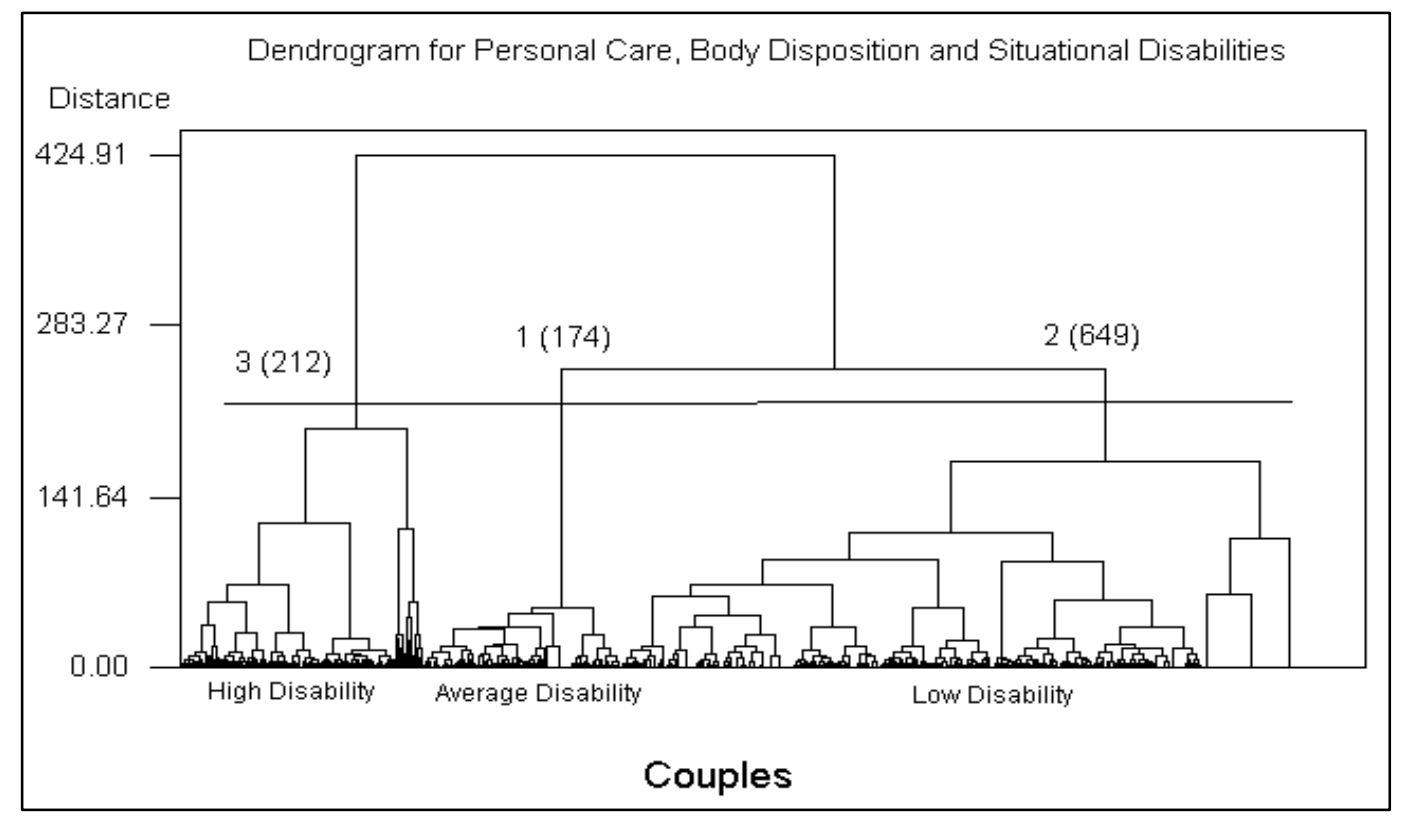

Figure 4. Dendrogram Showing the Clusters of Couples for Personal Care, Body Disposition, and Situational Disabilities.

Table 18. Characteristics of the Clusters of Couples on Personal Care, Body Disposition, and Situational Disabilities.

\begin{tabular}{|c|c|c|c|c|}
\hline Cluster $($ Size $\%)$ & Personal Care & Body Disposition & Situational & Overall \\
\hline $1(16.8 \%)$ & Low & Average & Average & Average \\
$2(62.7 \%)$ & Average & Low & Low & Low \\
$3(20.5 \%)$ & High & High & High & High \\
\hline
\end{tabular}

The question now arises what are the socio-economic variables associated with these clusters. We employed CDA on these clusters with the six socio-economic variables as predictors. However the method yielded no proper results as the score plot did not show any discrimination of clusters. Therefore we employed nominal logistic regression with the six variables as predictors. We did not apply the ordinal logistic regression as the ranking of these three clusters is not completely supported by all the 20 variables included in this analysis. The application of nominal logistic regression has been executed by considering the cluster 3 as the reference group. The cluster 3 is chosen as reference group, because this is the vulnerable cluster, which has the most affected or disabled people and to be compared.

The nominal variable 'Personal care and related disability' has been categorized as 'High', 'Average', and 'Low' as shown in the above table. Table 19 describes the results of nominal logistic regression. This table reveals that there is insufficient evidence to conclude that the changes in income and energy consumption have affected the low disabled people to become highly disabled. However, it is evident that the changes in occupational levels, expenditure, and protein consumption have affected the low disabled people to become high disabled. In fact, the declines in occupational levels and protein consumption had more impact on these changes.

If we consider the logit of average disability against high disability, there is insufficient evidence to conclude that the changes in occupational levels, income, and energy consumption have affected the people to become high disabled. However, it is clear that the changes in expenditure and protein consumption have affected the average disabled people to become high disabled. In general we can conclude that the higher disability in personal care, body disposition, and situational disabilities have been caused or stimulated by the decline of occupational levels, household expenditure, and protein consumption. 
Table 19. Nominal Logistic Regression Table for Personal Care and Related Disabilities against Socio-Economic Variables.

\begin{tabular}{|c|c|c|c|c|c|}
\hline Predictor & Coef & StDev & $\mathrm{Z}$ & $\mathrm{P}$ & Odds Ratio \\
\hline \multicolumn{6}{|c|}{ Logit 1: (Average Disability / High Disability) } \\
\hline Constant & 0.5587 & 0.3658 & 1.53 & 0.127 & \\
\hline OcLeH & 0.018383 & 0.008337 & 2.21 & 0.027 & 1.02 \\
\hline OcLeW & 0.03171 & 0.01271 & 2.49 & 0.013 & 1.03 \\
\hline PCExp & -0.0008301 & 0.00024 & -3.46 & 0.001 & 1 \\
\hline PCInc & 0.0000447 & 0.000113 & 0.39 & 0.693 & 1 \\
\hline PCEnC & -0.0000202 & 0.000217 & -0.09 & 0.926 & 1 \\
\hline PCPrC & 0.013593 & 0.007902 & 1.72 & 0.085 & 1.01 \\
\hline \multicolumn{6}{|c|}{ Logit 2: (Low Disability / High Disability) } \\
\hline Constant & -0.7847 & 0.4689 & -1.67 & 0.094 & \\
\hline OcLeH & -0.00834 & 0.01141 & -0.73 & 0.465 & 0.99 \\
\hline OcLeW & 0.00983 & 0.01852 & 0.53 & 0.596 & 1.01 \\
\hline PCExp & -0.0008903 & 0.000328 & -2.71 & 0.007 & 1 \\
\hline PCInc & 0.0000697 & 0.000148 & 0.47 & 0.639 & 1 \\
\hline PCEnC & 0.0001431 & 0.000259 & 0.55 & 0.58 & 1 \\
\hline PCPrC & 0.017421 & 0.009434 & 1.85 & 0.065 & 1.02 \\
\hline
\end{tabular}

Test all slopes are zero: $\mathrm{G}=42.905, \mathrm{DF}=12, \mathrm{P}-$ Value $<0.0001$

Goodness-of-Fit Tests : Chi-Square $=2055.524, \mathrm{DF}=2054, \mathrm{P}=0.486$

\section{Findings and Conclusions}

\subsection{Nature of Physical Disabilities}

If the general disability in terms of mobility is concerned, it seems that about $95 \%$ and $91 \%$ of the husbands and wives respectively can walk properly. About $94 \%$ of the husbands and $25 \%$ of the wives respectively can do cycling. The women do not do cycling as much as men, may be due to cultural barriers on the habits of cycling.

The behavior disability is described by self-awareness, identification, personal safety, knowledge acquisition, family role and occupation role. It is found that about $61 \%$ of the husbands and $80 \%$ of the wives are disabled in 'self-awareness'. The percentages of husbands and wives who have disability in the other items are: identification 27 and 34, personal safety 17 and 56, knowledge acquisition 77 and 85 , family role 16 and 32 , and occupational role 17 and 76 respectively. We extracted four factors, which together explain $70.6 \%$ of the total variation of behavior disability. About $23 \%$ of the time the behavior disabilities of the persons are prevalent with their self-awareness and knowledge acquisition. The prevalence of behavior disability is about $16 \%$ of the times on their identification. Further the disabilities of personal safety, family role and occupational role of the persons also influence the behavior disability. The prevalent of this disability is about $18 \%$ and $14 \%$ for husbands and wives respectively.

The communication disability is described by understanding speech, talking, listening, visual tasks, writing, and symbolic communication. It is found that, about $34 \%$ of the husbands and $43 \%$ of the wives does not understand speech. The percentages of husbands and wives who have disability in the other items are: talking $3 \%$ and $4 \%$, listening $10 \%$ and $9 \%$, visual tasks $60 \%$ and $53 \%$, writing $58 \%$ and $57 \%$, and symbolic communication $72 \%$ and $87 \%$ respectively. This may not be solely by literacy, but may be due to the destructed socio-cultural and political environment by the war. We extracted five factors where about $22 \%$ of the time the disabilities of the persons are prevalent with their disability on writing and symbolic communication. The prevalent of communication disability is about $13 \%$ of the times on visual tasks and about $12 \%$ of the times with their inability in understanding speech. Further the disabilities in talking and listening of the persons also influence about $13 \%$ and $12 \%$ of the times.

The personal care disability is described by excretory, bathing, personal hygiene, clothing, feeding, and transfer. It is found that, about $21 \%$ of the husbands and $17 \%$ of the wives have difficulties in 'excretory'. Similarly the percentages of other disabilities for husbands and wives are about $12 \%$ and $8 \%$ for bathing, $1 \%$ and $1 \%$ for personal hygiene, $3 \%$ and $1 \%$ for clothing, $1 \%$ and $1 \%$ for feeding, and $36 \%$ and $26 \%$ for transfer respectively. We extracted four factors where about $38 \%$ of the times the personal care disabilities of the persons are prevalent with their disability on personal hygiene, clothing, and feeding. Further, about $35 \%$ of the times this is prevalent with their disability on excretory, bathing, and transfer.

The body disposition disability is described by subsistence activity and household activity. It is found that about $20 \%$ of the husbands and $18 \%$ of the wives have disability in subsistence activity. About $66 \%$ and $41 \%$ of 
them have disability in household activity. The situational disability is described by dependence and environment. We found that about $49 \%$ of the husbands and $39 \%$ of the wives have disability in dependence. Similarly about $37 \%$ and $36 \%$ of them have disability in environment. We extracted three factors on the combined dimensions of body disposition and situational disabilities. The results reveal that about $45 \%$ of the times the disabilities of the persons are prevalent with their disability on household activity, dependence and environment. Further, about 19\% of the times the disabilities of the persons are prevalent with their disability on subsistence activity.

To explore the more general, but gender specific nature, all the variables for husbands were considered separately and similarly for wife. We found eight-factor formation sensible for both husbands and wives. The extracted eight disability factors have been formed by a more meaningful manner and it is also slightly different from the factor formations within the dimensions considered with their spouses. The gender difference in physical disabilities could be compared if we consider the formations of factors for husbands and wives. If we examine the factors, it is clear that almost all the factors are identical except with some minor differences regarding disabilities in understanding speech and subsistence activity.

\subsection{Socio-Economic Impacts}

It is found that the behavior disability of husband is highly correlated with his all other physical disabilities and reasonably associated with his wife's behavior and communication disabilities. Similarly, wife's behavior disability is highly correlated with her communication disability and her husband's communication, body disposition, and situational disabilities. In addition, the husbands and wives seem to have reasonable common communication, body disposition, and situational disabilities. It is also clear that their personal care, body disposition and situational disabilities are reasonably correlated.

It is also clear that the associations of physical disabilities between husbands and wives have been mainly due to their common disabilities in identification, knowledge acquisition, symbolic communication, and subsistence activities. If we consider the associations of different disabilities of husbands it is clear that the disability in knowledge acquisition further enable the disability in symbolic communication. This is also true for wives. Husband's disabilities in personal safety and personal hygiene are interrelated and family role is related to his subsistence activities. Further, his body transfer and household activities are also related. Regarding the associations of wife's disabilities, transfer and household activities are related.

The socio-economic variables: 'occupation of parents', 'per capita income and expenditure', 'per capita energy and protein consumption' were considered with the above disabilities. Some homogeneous clusters of couples in relation to 'Behavioral and communication disabilities', 'Personal care and related disabilities', 'Psychological disorders', and 'Physiological and related disorders' were found. In the case of behavior and communication disabilities, we found two distinct groups of couples who possess different disabilities. We found that the cluster of $58 \%$ of the couples is influenced by the higher degree of disabilities in self-awareness, knowledge acquisition, understanding speech, and symbolic communication of husbands and in identification, understanding speech, and listening of wives. These characteristics are low in the other cluster. Other disabilities do not seem to dominate in the discrimination of these two clusters. We also found that higher occupational level of husbands is strongly associated with the cluster of the couples of low behavior and communication disabilities. Higher occupational level of wives, higher per capita income and expenditure also seems to have reasonable association.

If we consider the other physical disabilities, we found the existence of three clusters. We found that about $20 \%$ of the people have higher and about $17 \%$ of the people have average personal care and related disabilities. The rest of the people have mild or no personal care and related disabilities. We found that there is insufficient evidence to conclude that the changes in income and energy consumption have affected the low disabled people to become high disabled. However, it is evident that the changes in occupational levels, expenditure, and protein consumption have affected the low disabled people to become high disabled. In fact, the declines in occupational levels and protein consumption have made these changes. If we consider average disability against high disability, there is insufficient evidence to conclude that the changes in occupational levels, income, and energy consumption have affected the people to become high disabled. However, it is clear that the changes in expenditure and protein consumption have affected the average disabled people to become high disabled. In general we can conclude that the higher disability in personal care, body disposition, and situational disabilities have been caused or stimulated by the decline of occupational levels, household expenditure, and protein consumption.

\section{REFERENCES}

[1] Elankumaran, C. (1999) JSEHS - Jaffna Socio-Economic Health Study, Field Data Collection Survey, Ph.D research project on Medical and Economic Statistics, Funded by University of Jaffna and approved by Department of Health, Jaffna.

[2] Elankumaran,C. (2001) A Quantitative Analysis of the Relationship between Soci-Economic Status and Health Conditions of the people of Jaffna Peninsula, Sri Lanka with 
special reference to the Impacts of Exodus 1995, Ph.D thesis, University of Jaffna, Un-published.

[3] Elankumaran,C. and Sivagnanasundram,C. (2002) Socio-Economic and Health Impacts of Exodus 1995 in Jaffna Peninsula, Proceedings of the Sixth International Medical Congress, University of Peradeniya, 2002 Dec 13-15.

[4] WHO (1980) International Classification of Impairments, Disabilities, and Handicaps, A manual of classification relating to the consequence of Diseases, World Health Organization, Geneva.

[5] Sivarajah,V. (1997) War and Health in Jaffna, Sectional Chairman's address -1997, Medical Sciences Section, Proceedings of the Jaffna Science Association, University of Jaffna, 1998, pp39-51.

[6] Somasundram,D.J., Sivayogan,S. \& Muhunthan, N. (1995) War Trauma in a Civilian Population, Ninth annual session of Jaffna Medical Association, Jaffna Medical Journal, Vol.25, No.1, p39.

[7] Ebrahim,S., Nouri,F., and Barer,D. (1985) Measuring disability after a stroke. Journal of Epidemiology and Community Health. Vol.39, pp86-89.

[8] Thorslund,M., Norstrom,T., and Wernberg,K. (1991) The utilization of home help in sweden - A Multivariate analysis. The Gerontologist. Vol.31, No.1, pp116-119.

[9] Norstrom,T. and Thorslund,M. (1991) The structure of IADL and ADL measures - Some findings from a Swedish study. Age and Aging, Vol.20, pp23-28.

[10] Kai,I., Ohi,G., Kobayachi,Y., Ishizaki,T., Hisata,M., and Kiuchi,M. (1991) Quality of Life : A possible Health index for the elderly. Asia-Pacific Journal of Public Health. Vol.5, No.3, pp221-227.

[11] Kempen,G.I.J.M. and Suurmeijer,P.B.M. (1991) Factors influencing professional home care utilization among the elderly. Social Science and Medicine. Vol.32, No.1, pp77-81.

[12] McLennon,W. (1990) Disability and Handicap in Australia, 1988. Australian Bureau of Statistics, Catalogue No.4120.0. October 1990.

[13] Janet Reis (1988) A factor analysis of a compound measure of social support. Journal of Clinical Psychology, November 1988, Vol.44, No.6, pp876-890.

[14] Jagger,C., Clarke,M., and Davies,R.A. (1986) The elderly at home: Indices of Disability. Journal of Epidemiology and Community Health. Vol.40, pp139-142. 